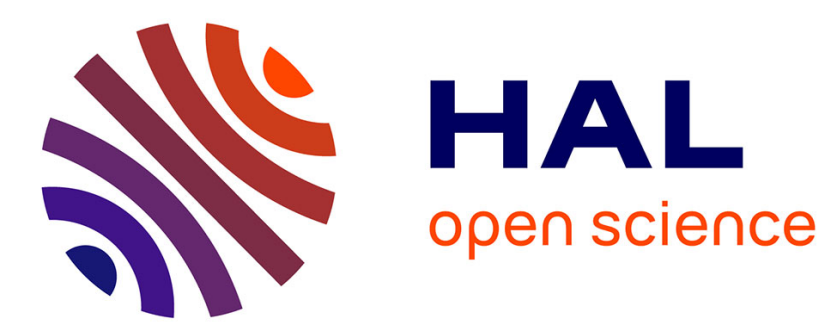

\title{
Simulations and Experiments of a Nonlinear Aircraft Braking System With Physical Dispersion
}

Fabrice Chevillot, Jean-Jacques Sinou, Nicolas Hardouin, Louis Jézéquel

\section{To cite this version:}

Fabrice Chevillot, Jean-Jacques Sinou, Nicolas Hardouin, Louis Jézéquel. Simulations and Experiments of a Nonlinear Aircraft Braking System With Physical Dispersion. Journal of Vibration and Acoustics, 2010, 132 (4), pp.041010. 10.1115/1.4000982 . hal-00625101

\section{HAL Id: hal-00625101 \\ https://hal.science/hal-00625101}

Submitted on 30 May 2018

HAL is a multi-disciplinary open access archive for the deposit and dissemination of scientific research documents, whether they are published or not. The documents may come from teaching and research institutions in France or abroad, or from public or private research centers.
L'archive ouverte pluridisciplinaire HAL, est destinée au dépôt et à la diffusion de documents scientifiques de niveau recherche, publiés ou non, émanant des établissements d'enseignement et de recherche français ou étrangers, des laboratoires publics ou privés. 


\title{
Simulations and Experiments of a Nonlinear Aircraft Braking System With Physical Dispersion
}

\author{
Fabrice Chevillot ${ }^{\mathrm{a}, \mathrm{b}}$, Jean-Jacques Sinou ${ }^{\mathrm{a}}$, Nicolas Hardouin ${ }^{\mathrm{b}}$, Louis Jézéquel ${ }^{\mathrm{a}}$ \\ aLaboratoire de Tribologie et Dynamique des Systèmes, Equipe D2S, UMR-CNRS 5513, Ecole Centrale de Lyon, 36 Avenue Guy de \\ Collongue, 69134 Ecully, France \\ ${ }^{b}$ Messier-Bugatti-Safran Group, Aircraft Braking Division, Zone Aéronautique Louis Bréguet, BP 40, 78140 Vélizy-Villacoublay, France
}

\begin{abstract}
This paper deals with the simulation of nonlinear vibration induced by friction in an aircraft braking system. Experimental tests reveal that in similar experimental conditions the mechanism can generate vibrations of various amplitudes. The aim of this study is to simulate the behavior of the brake by taking into account the dispersion of parameters, which produce the variability of the response. A nonlinear model of the brake is pre-sented. The time-history response is obtained by integration of the full set of nonlinear dynamic equations. Based on experimental results, the dispersions of the coefficient of friction and of the damping configuration are introduced. Monte Carlo simulations are performed and show a very good agreement with the experimental results.
\end{abstract}

\section{Introduction}

In aircraft braking systems, vibrations under $1000 \mathrm{~Hz}$ appear during the braking phase. These are caused by friction-induced self-generated instabilities, which are a cause of concern in a wide variety of mechanical systems, especially in transports. In the automotive industry the phenomenon of brake squeal is well known of end-users because of the noise produced. However, it still retains attention of a lot of investigations [1-4]. In the rail industry it is an issue for the same reasons [5,6]. In aircraft brakes Liu et al. $[7,8]$ and Gordon [9] are among the first to describe frictioninduced vibrations and to present the equations of motion for the phenomenon known as squeal. The whirl phenomenon is then studied by Travis [10] and Hagler [11], and completed by the work of Sinou et al. $[12,13]$. A complete model for the simulation of whirl and squeal instabilities is finally presented by Chevillot et al. $[14,15]$.

The study of a vibration problem requires two steps. In a first step, a stability analysis is performed around the operating point by linearization of the nonlinear equations. The nature of the complex eigenvalues of the Jacobian matrix provides information on the local stability of the operating point. It allows determining if the equilibrium is linearly stable or not: If unstable, instability corresponds to a supercritical Hopf bifurcation. The stability analysis also gives the nature of the instabilities by plotting their analytical deformation. This first step is fast to compute. For this reason, this approach is especially useful to evaluate the effects of parameters on the stability. In that way, modifications in the design can be found to make the equilibrium stable. However, if the equilibrium is unstable, the stability analysis is unable to give information on the nonlinear dynamical behavior. As instability is the result of a supercritical Hopf bifurcation, a stable periodic orbit will appear. Then, the direct approach consists in integrating numerically the equations of motion around the operating point to calculate the amplitude of the stable orbit. Although this procedure can be time-consuming, especially if nonlinear models with many degrees of freedom are employed, it is necessary to know the entire time-history response of the system [15]. In that context, the use of a mechanical model with a few degrees of freedom is very suitable.

The first part of this paper presents the working of an aircraft braking system and describes the test performed to record the dynamical behavior of the brake in working conditions. Then the 


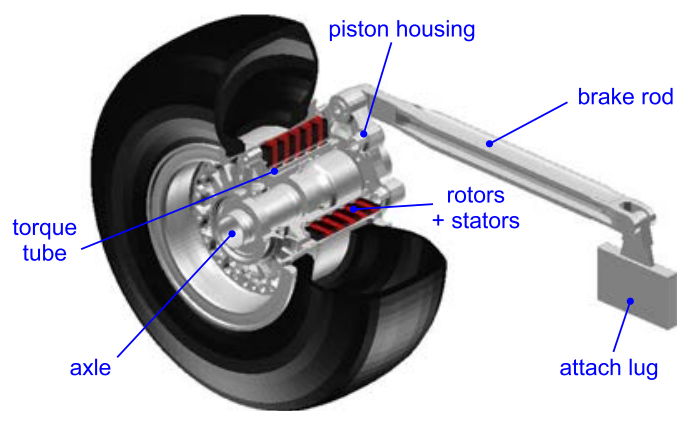

Fig. 1 Schematic model of the brake system

phenomenological model developed by Chevillot et al. in Refs. $[14,15]$ is briefly described. A stability analysis is made in order to determine the nature of the instabilities. They are compared with the experimental instabilities recorded during tests. Next, timehistory responses are computed in a particular case to evaluate the efficiency of the model to reproduce complex transient vibrations. Finally, with the statistical laws for the coefficient of friction and for the damping, a Monte Carlo simulation is computed in order to reproduce the variability of the dynamical behavior observed when a series of tests is performed.

\section{Aircraft Brake Description}

2.1 Experimental Approach. An aircraft braking system is shown in Fig. 1 where the rotating parts are given in a threequarter section view. The brake is attached to the landing gear of the aircraft through a torque take-out rod. The core of the brake is composed of a stack of rotating and stationary disks: The rotating disks - the rotors - are engaged by the wheel, while the stationary disks - the stators - are linked to the torque tube, which is interdependent of the piston housing. The working principle is illustrated in Fig. 2. During braking, the rotors and stators are squeezed together by pressure in the hydraulic pistons. Torque is produced by friction forces generated at the rubbing interfaces between the disks. Aircraft slowing and stopping is therefore the result of the conversion of the kinetic energy of the plane into heat in the brake.

The evaluation of the dynamical behavior of the brake under working conditions is performed with the help of dynamic tests illustrated in Fig. 3. The brake is set on a roadwheel dynamometer, which simulates the inertia of the aircraft. The roadwheel dynamometer is brought up to speed, then pressure is introduced in the hydraulic pistons to activate the brake by compression of the heat stack. The test ends when the roadwheel dynamometer stops. A series of tests with a fully instrumented aircraft brake is performed in order to record the dynamical behavior of the system

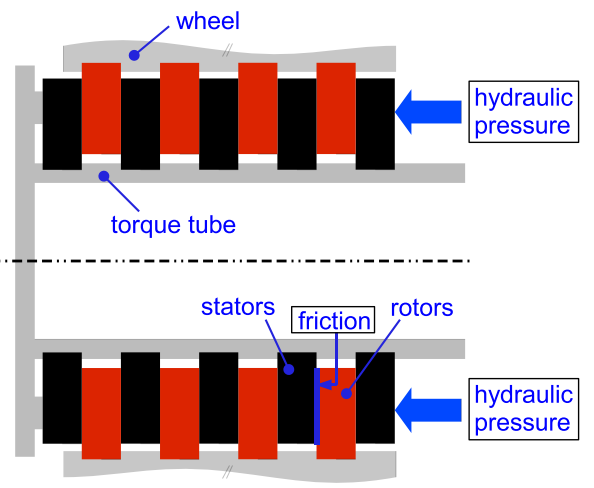

Fig. 2 Working principle

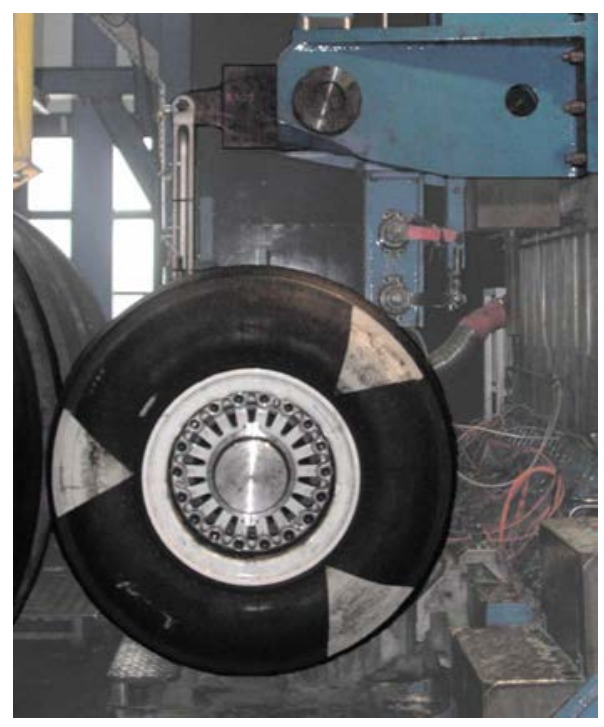

Fig. 3 View of a dynamic test

during braking. The accelerometers' locations on each instrumented part are shown in Fig. 4. The pressure introduced and the torque produced are also recorded.

Tests allow identifying two main complex nonlinear phenomena: squeal and whirl. Both appear at low/middle frequency (in the $0-1000 \mathrm{~Hz}$ range) and can damage the integrity of the brake when the amplitude of oscillations is substantial. On the one hand, squeal is defined as torsional vibrations of nonrotating brake parts around the axle; on the other hand, whirl describes a motion of the end of the torque tube around the axle accompanied by unphased pumping of the brake pistons. The experimental deformations of these two phenomena are given in Fig. 5. The markers from 1 to 6 indicate the evolution of the deformation of the brake for one period. The shaded lines define the static position.

2.2 Phenomenological Model. A phenomenological model is built to reproduce whirl and squeal instabilities [14]. The brake, the brake rod, and the attachment lug are modeled with 70 degrees of freedom (see Fig. 6). The multistage brake is represented by a single rotor and stator with an effective brake friction coefficient of $\mu_{\mathrm{eq}}=2 N_{\text {rotor }} \mu$, where $N_{\text {rotor }}$ is the number of rotors and $\mu$ is the coefficient of friction between two disks. A Coulomb approach is used to model the friction. It is assumed that each rubbing interface is equivalent and that the friction coefficient is uniform on the surface. Moreover contact and sliding states are supposed to be permanent. Experimental tests $[12,14]$ show that the nonlinear behavior of the disk stack in compression is nonlinear. The loadcompression relationship is introduced analytically by a third or-

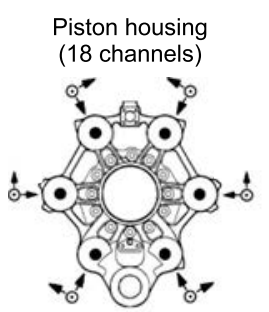

$$
\begin{aligned}
& \text { Torque plate } \\
& \text { (12 channels) }
\end{aligned}
$$

Attach lug
$(2$ channels $)$
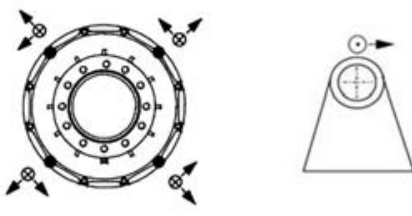

Axle (7 channels)

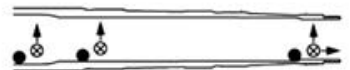

Brake rod (12 channels)

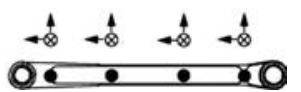

Fig. 4 Accelerometers' locations 
(a)
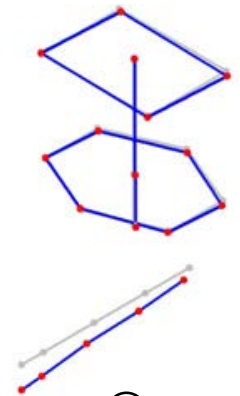

(4)
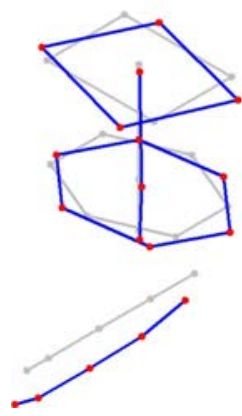

(b)

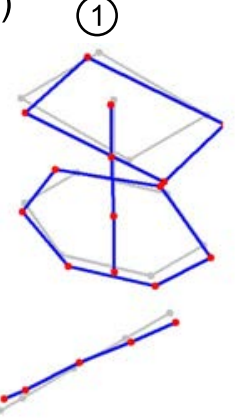

(4)
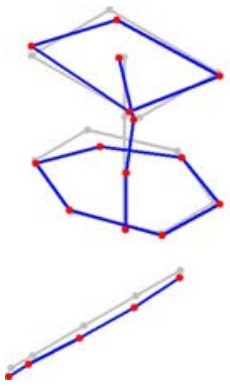

Fig. 5 Experimental deformations: (a) squeal and (b) whir
(5)

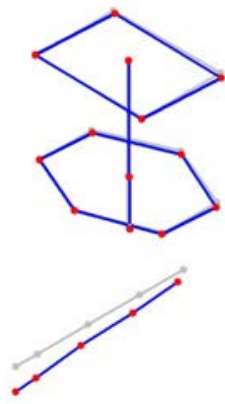

(2)
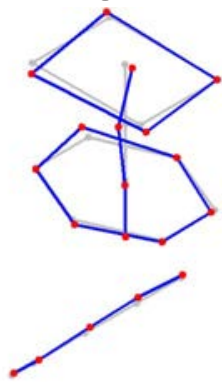

(5)
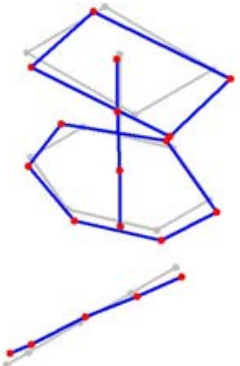

Description:

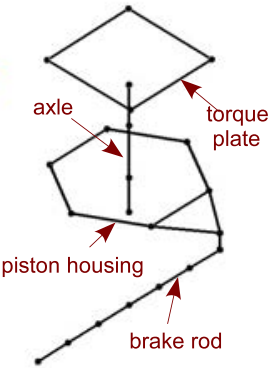

specified for each mode of the structure, in the modal basis. The diagonal damping matrix thus built is then projected onto the basis of the degrees of freedom to create the structural damping matrix C of Eq. (1).

A stability analysis is then performed in order to calculate the modes of the system and the mode-coupling instabilities, if there are any. The detailed method can be found in Ref. [12] or Ref. [14] and is briefly given below.

The stability analysis is conducted around the equilibrium point $\mathbf{x}_{0}$. It is given by solving the nonlinear system defined by Eq. (1) in the static case

$$
\mathbf{K} \mathbf{x}_{0}=\mathbf{F}_{\mathrm{NL}}\left(\mathbf{x}_{0}\right)+\mathbf{F}_{\text {pressure }}+\mathbf{F}_{\text {tire }}
$$

The stability is then investigated around the equilibrium point for a small perturbation $\overline{\mathbf{x}}$ by linearizing the nonlinear contact forces

$$
\mathbf{M} \ddot{\overline{\mathbf{x}}}+\mathbf{C} \dot{\overline{\mathbf{x}}}+\left(\mathbf{K}-\left.\frac{\partial \mathbf{F}_{\mathrm{NL}}}{\partial \overline{\mathbf{x}}}\right|_{\mathbf{x}_{0}}\right) \overline{\mathbf{x}}=0
$$

The stability of the equilibrium is discussed by calculating the eigenvalues of the linear system given by Eq. (3). These are expressed as $\lambda=a+i b: b$ represents the angular frequency (i.e., the frequency by considering a factor of $2 \pi$ ), and a damping coefficient can be written by $\eta=-a /|\lambda|$. The equilibrium of the system is then unstable if at least one eigenvalue has a strictly positive real part (which is equivalent to a strictly negative damping coefficient).

A representation of the evolution of the frequencies versus the coefficient of friction in the complex plane is given in Fig. 7. It appears that between $0 \mathrm{~Hz}$ and $1000 \mathrm{~Hz}$ three instabilities are calculated: Each instability is the result of the coupling of two modes (one is unstable while the other one remains stable). The nature of the instabilities can be identified by plotting their analytical mode shapes given in Fig. 8: The first one is identified as a squeal instability and the second one as a whirl instability. A good agreement between the analytical deformations and the experimental deformations can be noticed. The phenomenological model is then able to reproduce the instabilities observed in tests. Concerning the third instability calculated by the stability analysis, it seems also to have whirl characteristics, but its frequency around $600 \mathrm{~Hz}$ is far from the frequency of the experimental whirl (around $250 \mathrm{~Hz}$ ). Because the stability has been performed without damping, Fig. 7 gives also information on the damping coefficients, which make the system stable or unstable. For example, if a damping of more than $15 \%$ is introduced on all the modes, then no vibration can occur. More precisely, 3\% of damping for the squeal instability, $15 \%$ for the whirl instability, and $12 \%$ for the third instability are sufficient to make the equilibrium stable. It should be noted that the squeal instability will appear only if a very small damping is present. For the whirl vibration, if there is damping under $15 \%$, oscillations should appear. That illustrates the fact that squeal seems more difficult to destabilize than whirl:

During tests only a few appearances of squeal have indeed been observed.

\section{Time-Frequency Analysis: The CWT}

The time-frequency analysis is a key step in the investigation of the information contained in a temporal signal. For a stationary signal, a fast Fourier transform (FFT) is a commonly used method. It transforms the signal from a time-based domain to a frequency-based domain. However, in the case of a nonstationary signal, the time-dependence of the frequency components can be an essential element in order to obtain good understanding of the behavior. To achieve this, methods are employed to generate timefrequency representations of the signal. For a nonstationary signal with sharp time-varying frequency components, the continuous wavelet transform (CWT) is an efficient method. Its applications 


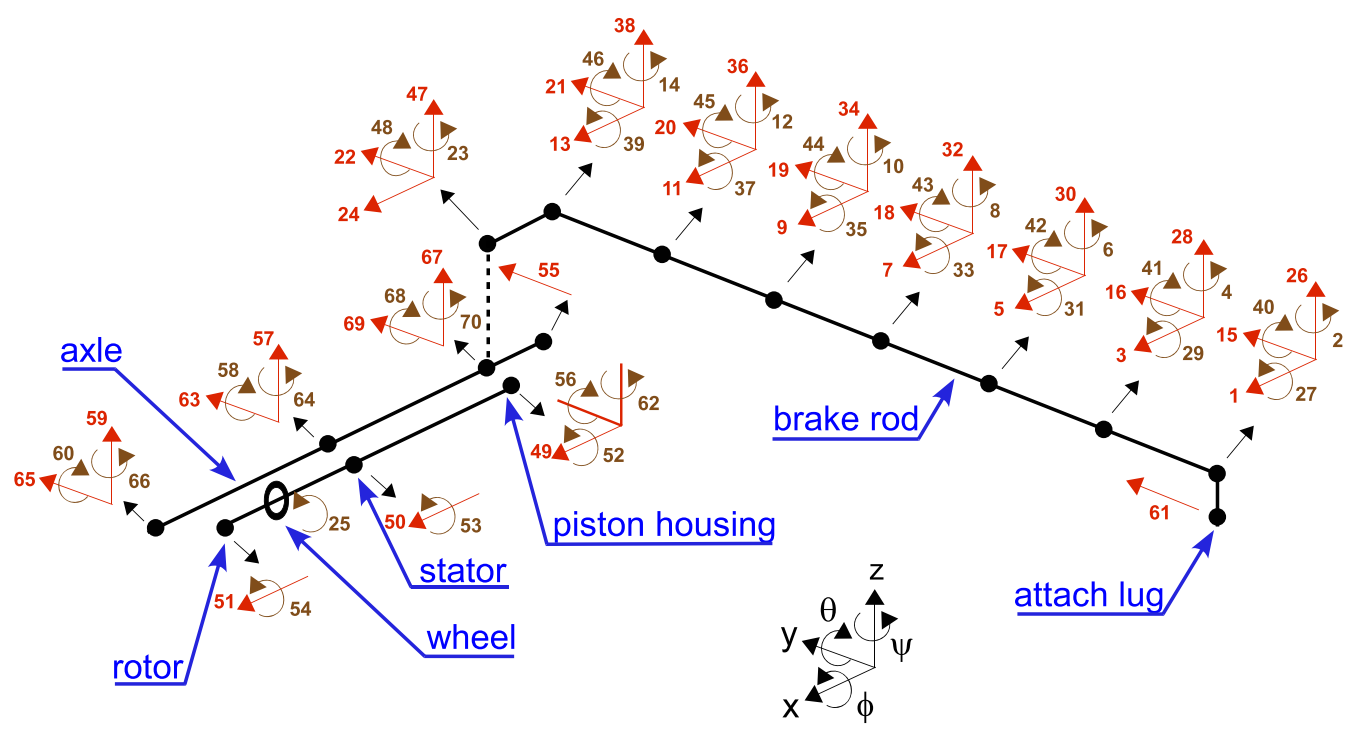

Fig. 670 degree-of-freedom model

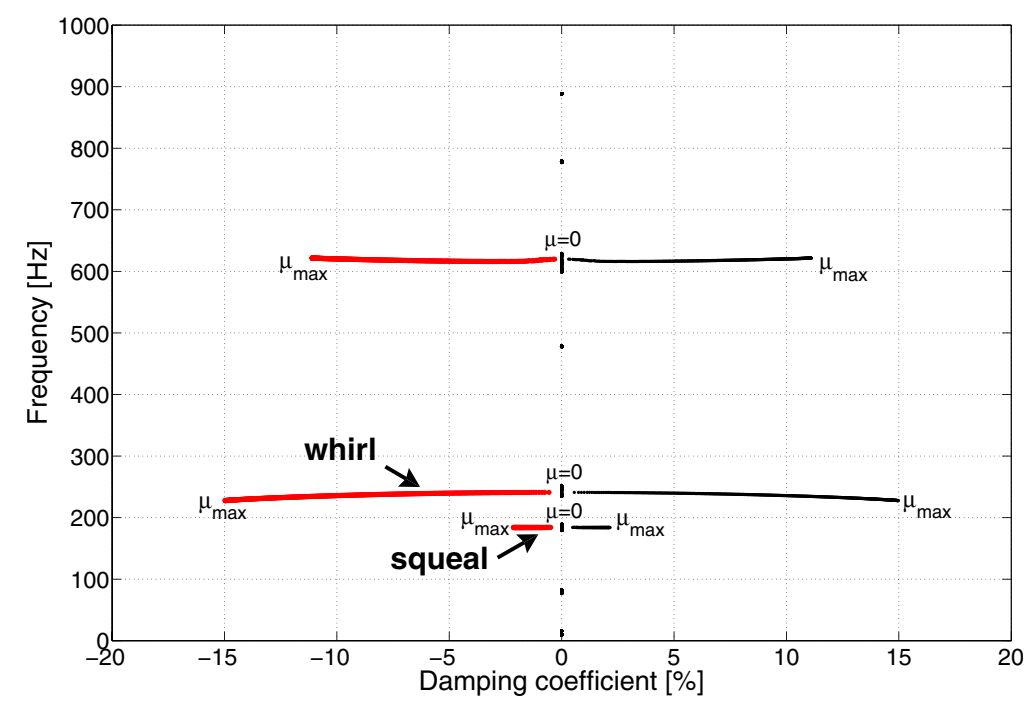

Fig. 7 Stability of the brake versus friction coefficient in the complex plane

are various; for instance, it can be employed to find the presence of a crack in rotors [16]. Some of CWT software includes code originally written by Torrence and Compo. ${ }^{2}$

Wavelet transforms first appeared in the 1980s with the work of Morlet [17]. The theoretical background can be found in Refs. [18-20]. A wavelet analysis starts by selecting an elementary wavelet function $\phi(t)$ whose decay is very fast and which fulfills the admissibility conditions $[19,20]$ : the mother wavelet. A family of functions (the wavelets) is then obtained by translation and dilatation of the mother wavelet

$$
\phi_{a, b}(t)=\frac{1}{\sqrt{a}} \phi\left(\frac{t-b}{a}\right)
$$

where $a$ is the dilatation or scale factor and $b$ is the time translation factor. The term $1 / \sqrt{a}$ ensures the energy normalization across the different scales.

The transform is then performed by projecting the signal $s(t)$ onto the wavelets, producing the CWT coefficients

\footnotetext{
${ }^{2}$ See http://paos.colorado.edu/research/wavelets/.
}

$$
\mathrm{CWT}_{\phi}^{s}(a, b)=\int_{-\infty}^{\infty} s(t) \phi_{a, b}^{*}(t) d t=\frac{1}{\sqrt{a}} \int_{-\infty}^{\infty} s(t) \phi^{*}\left(\frac{t-b}{a}\right) d t
$$

where ${ }^{*}$ denotes the complex conjugate.

The wavelet coefficients represent a measurement of the correlation of the dilated and shifted wavelet with the function $s(t)$. $\mathrm{CWT}_{\phi}^{s}(a, b)$ also express the local information on $s(t)$ at time $t$ $=b$ and frequency $f$ related to $1 / a$. A time-frequency representation is obtained by plotting the wavelet coefficients over time and frequency.

\section{Deterministic Simulation}

In this section, a specific experimental test is studied. The purpose is to evaluate the capability of the phenomenological model to reproduce the temporal behavior of the brake when vibration occurs. The time plots of the pressure and of the torque are given in Fig. 9. The response is composed of several steps. First the pressure is applied progressively, until it reaches the rated value $P_{R}$. At the same time the torque increases, without the appearance of vibration. Second, oscillations are developing with a large am- 
(a)

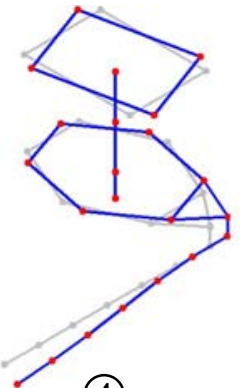

(4)

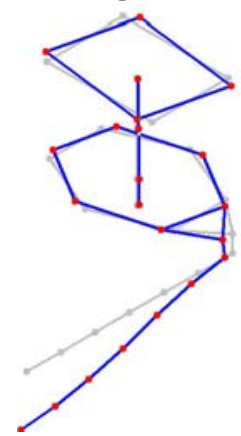

(b)

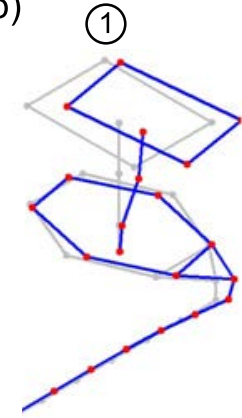

(4)
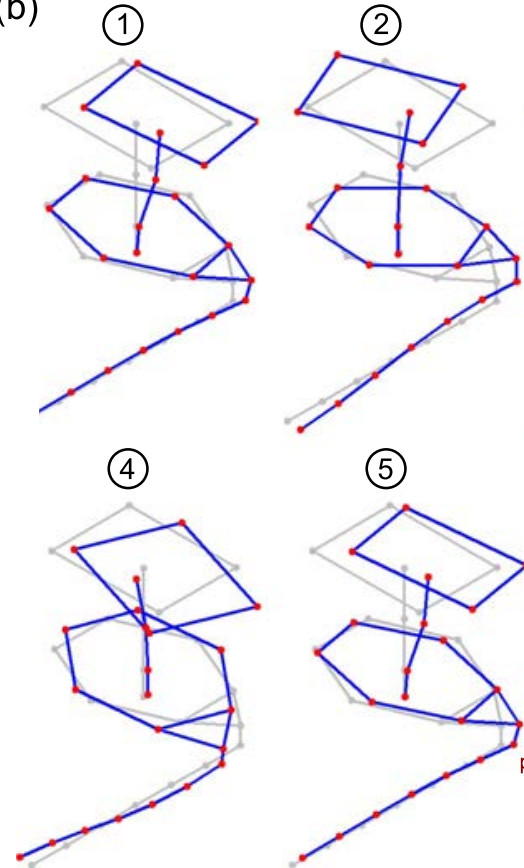

(5)
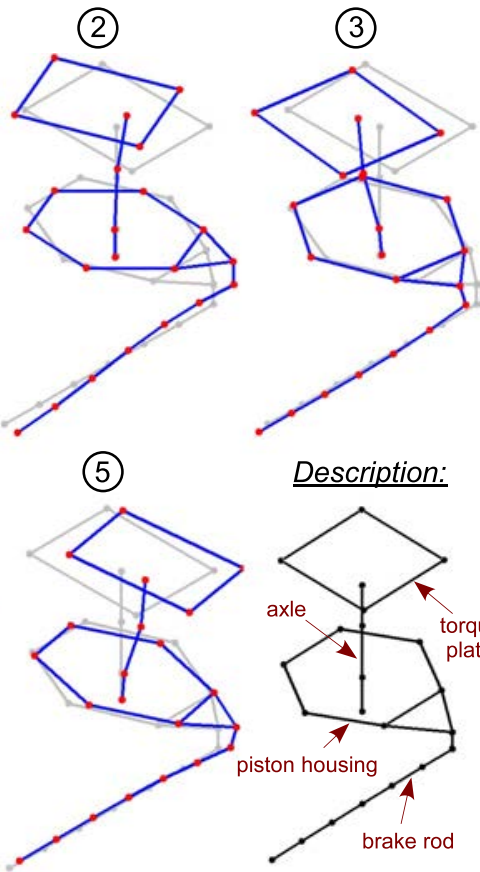

Description:

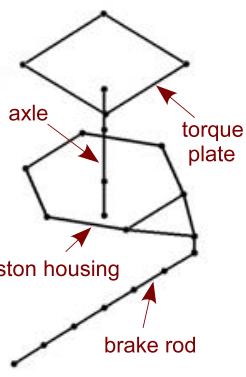

Fig. 8 Analytical deformations: (a) squeal and (b) whirl

plitude on the pressure and on the torque. Finally, after $7 \mathrm{~s}$ of braking, the oscillations have almost entirely disappeared. For this test, vibration occurs in the transient regime, while in the stationary regime almost no vibration remains. It should be noted that the evolution of the oscillations is not regular. The time plots reveal that there are abrupt changes at $t=1.5 \mathrm{~s}$ and $t=3 \mathrm{~s}$. It is then probable that several instabilities have appeared in the transient regime. A CWT of the pressure and of the torque given in Figs. $10(a)$ and $10(b)$ allows determining with precision the frequencies and their evolution versus time. It appears that two instabilities have occurred. The first one is measured at $260 \mathrm{~Hz}$ and appears briefly in the beginning of the braking. While this instability stops abruptly at $t=1.5 \mathrm{~s}$, a second instability at $180 \mathrm{~Hz}$ appears with large oscillation amplitudes. The latter only lasts about $1.5 \mathrm{~s}$ before the first instability appears again in place of the second instability. The CWT also shows the appearance of frequencies at 360 $\mathrm{Hz}$ and $520 \mathrm{~Hz}$, which are, in fact, overharmonics of the $180 \mathrm{~Hz}$ and $260 \mathrm{~Hz}$ : Their presence is the expression of the nonlinear behavior of the mechanism. The drawing of the experimental deformation of the brake when vibration occurs allows identifying the instabilities as a whirl vibration for the first one and a squeal vibration for the second one. This record is a good case of study because oscillations with a large amplitude occur in the transient regime. Moreover the appearance of two instabilities with a strong interaction is very interesting.

The simulation of a braking requires in inputs the evolutions of the pressure and of the coefficient of friction. Concerning the pressure it is simply introduced with a slope to reach the rated value $P_{R}$ (cf. Fig. 11(b)). For the coefficient of friction, it is calculated by the relation that links the pressure $P(t)$ to the torque $T(t)$, with the assumption that there are no friction forces somewhere else than between the disks

$$
T(t)=2 N_{\text {rotor }} \mu(t) P(t) N_{\text {piston }} S_{\text {piston }} \frac{2}{3}\left(\frac{R e^{3}-R i^{3}}{R e^{2}-R i^{2}}\right)
$$

where $N_{\text {rotor }}$ is the number of rotors, $N_{\text {piston }}$ is the number of pistons, $S_{\text {piston }}$ is the surface of a piston, and $R e$ and $R i$ are, respectively, the outer and inner radii of the disks.

The friction law obtained is given in Fig. 11(a). The shape can be explained by tribological properties of the heat sink material. To perform the simulation, the damping configuration is still missing. The response of the brake is indeed very dependent on the damping introduced. But the measurement of the damping of a complex mechanical system is very difficult, or even impossible in practice. The idea is then to perform simulations step by step by changing the damping coefficients until getting the desired response. Figure 12 shows the torque simulated with a modal damping coefficient of $0.3 \%$ for the squeal instability and $8 \%$ for the whirl instability. Because the hydraulic equations are not taken into account, the model is not capable of simulating the oscillations of the pressure. But it has no effect on the mechanical deformation of the brake. Concerning the third instability revealed by the stability analysis (see Fig. 7), 10\% of damping is introduced to prevent it from appearing. This value is arbitrary, as well as that of $1 \%$ used for all the other modes not involved in modecoupling instabilities. The time plot shows that oscillations appear in the transient regime. Their amplitude is quite similar to that of the experimental record and the changes in amplitude let us think that several instabilities are present. This intuition is confirmed by the CWT of the torque given in Fig. 13. The response is indeed composed of two main frequencies at $184 \mathrm{~Hz}$ and $240 \mathrm{~Hz}$. The first one corresponds to the squeal vibration and the second one to the whirl vibration. The overharmonics of squeal and whirl may be noticed: squeal $2 \mathrm{X}$ at $360 \mathrm{~Hz}$ and whirl $2 \mathrm{X}$ at $520 \mathrm{~Hz}$. In addition, there are combinations of the frequencies of squeal and whirl: squeal 2X-whirl at $128 \mathrm{~Hz}$, whirl 2X-squeal at $296 \mathrm{~Hz}$, and squeal+whirl at $424 \mathrm{~Hz}$. There exist only in the short intervals where both squeal and whirl are present. These combinations may also be noticed in the experimental CWT in the transition between whirl and squeal at $t \in\left[\begin{array}{ll}1 & 1.2\end{array}\right] \mathrm{s}$.

In a first time, whirl appears for about $1 \mathrm{~s}$. Then oscillations of squeal increase while the oscillations of whirl stop. The squeal vibration lasts about $2 \mathrm{~s}$ and finally whirl appears again. A better comparison of the transient behaviors is possible with Fig. 14, which shows the appearances of whirl, squeal, whirl $2 X$, and squeal $2 \mathrm{X}$ in the first $4 \mathrm{~s}$ of braking. There is a good agreement, although slight differences may be noticed. In the test, the transition between whirl and squeal is more abrupt than in the simulation. Therefore, the interval where the two instabilities are simultaneously present is longer in the simulation, which results in combinations of frequencies more pronounced. In the stationary regime, it may be noted that the second harmonic of whirl re- 

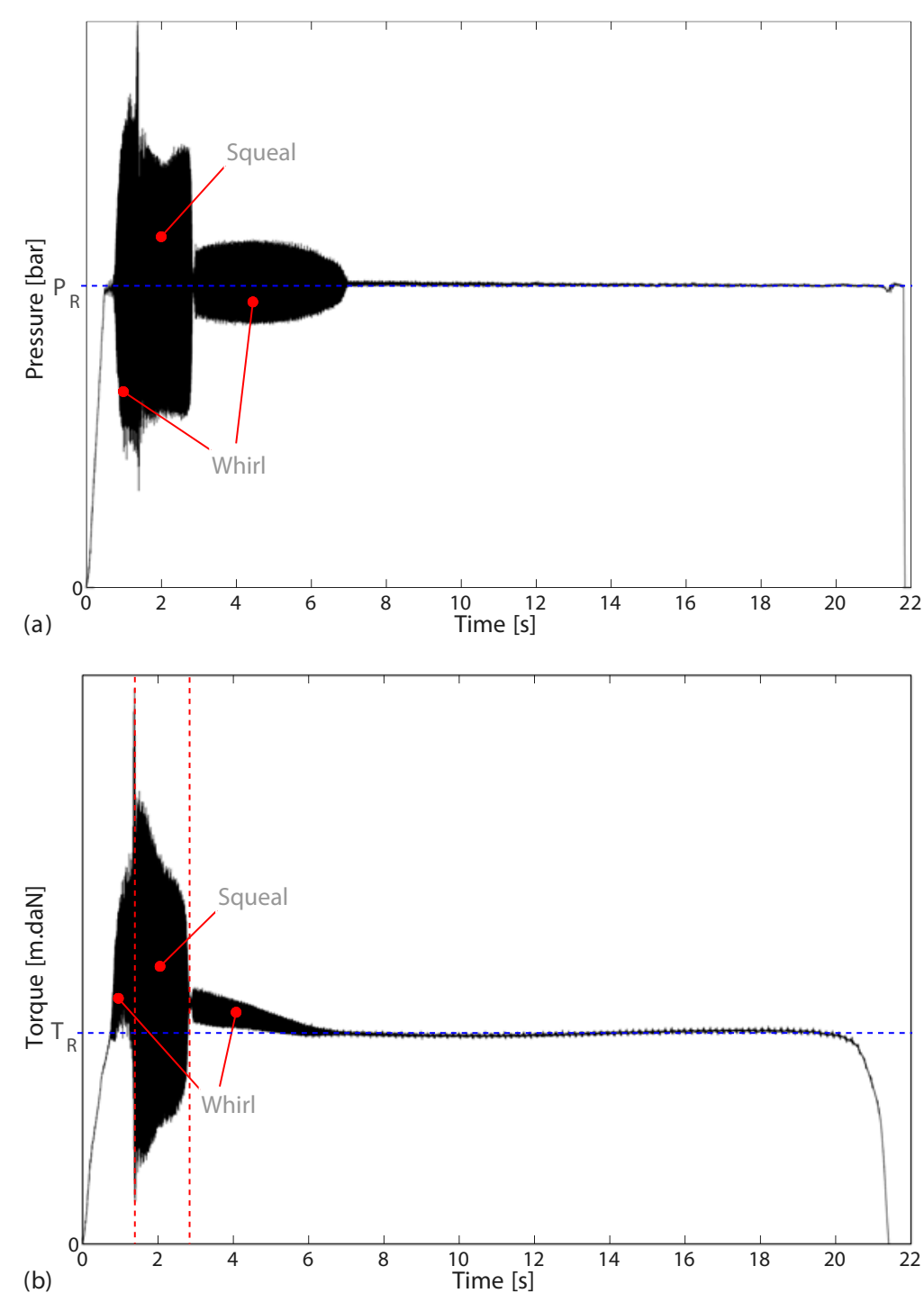

Fig. 9 Experimental record: (a) time plot of the pressure and (b) time plot of the torque

mains, while it has disappeared in the test. In a general way, the overharmonics seem a little more pronounced in the simulation.

A very good agreement between the simulated and experimental behaviors is obtained. This example shows the efficiency of the phenomenological model to simulate the transient nonlinear vibrations observed during tests.

\section{Statistical Simulation}

When a series of tests is performed in order to record the dynamical behavior of the brake, it appears that the response of the system can differ between two tests although the external conditions are apparently identical. The effect of this dispersion is illustrated in Fig. 15(a), which shows the maximum amplitude of the acceleration measured on the piston housing for 112 tests performed. In this series of tests only the whirl vibration at around $260 \mathrm{~Hz}$ appeared; there is no occurrence of squeal.

In Fig. 15(b) is given a pie chart, which shows the distribution of the maximum acceleration. It reveals that for more than half of the tests no vibration or a vibration with a very low amplitude (under 10\% of Gmax) has appeared. And when the whirl has developed, the figure shows a distribution quite regular between the different levels. It is then obvious that internal changes occurred from a test to another. Therefore in order to simulate with a good accuracy the dynamical behavior of the brake when a series of tests is performed, these internal changes have to be identified and taken into account.

The heat sink material has tribological characteristics, which make the coefficient of friction highly dependent on the temperature. Because of the succession of tests, the warming of the brake can be different between two stops. As a result the coefficient of friction will slightly vary. Each test performed also wears out the rubbing surfaces. From this point of view two successive tests are not equivalent. That can explain variations in the coefficient of friction. For each test, with the records of the pressure and of the torque, the coefficient of friction is calculated. In Fig. 16(a) are given the time plots of the coefficient of friction of the 112 stops performed. The mean value $\bar{\mu}$ and the standard deviation $\sigma$ give information on the variability of the coefficient of friction. It should be noted that the curves are well centered around the mean value and that all curves are approximately obtained by a translation of the mean curve. For this reason, it seems appropriate to consider a Gaussian distribution to represent the variability of the coefficient of friction. Figure 16(b) shows a Monte Carlo simulation with 200 friction laws. To avoid very small or very high value of the coefficient of friction, which would not be physical, the Gaussian distribution is truncated at $\pm 2.5 \sigma$. 

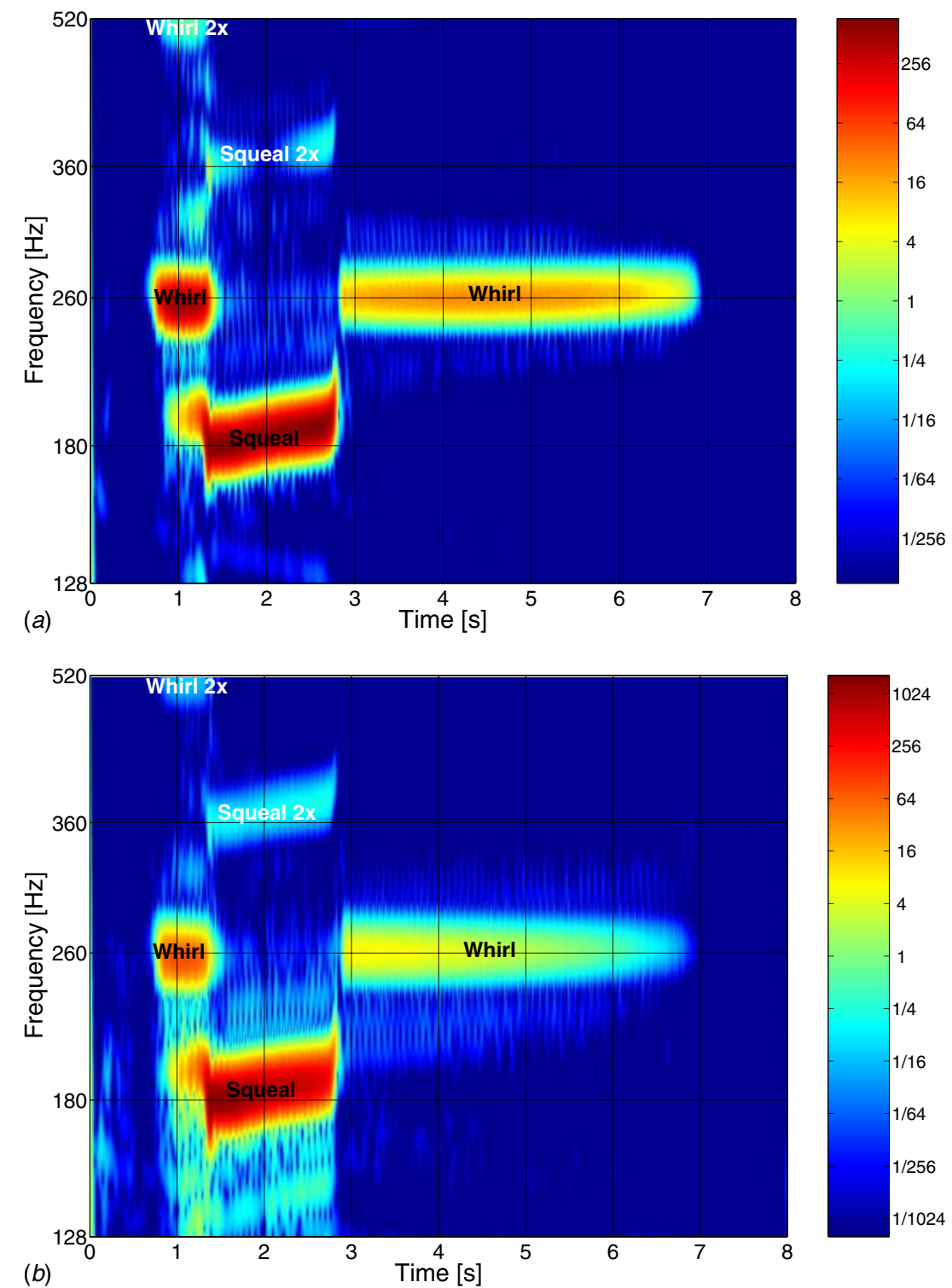

Fig. 10 Experimental record: (a) CWT of the pressure and (b) CWT of the torque

The succession of tests, with variations in the temperature and the progressive wear of the disks, has also an effect on the damping of the brake. However, the measurement of the damping configuration is not possible. A few deterministic simulations have been performed to evaluate the range and variability of the damping of the whirl. Tests with extreme behaviors (very large and very small amplitudes of vibration) and with medium behaviors have, in particular, been chosen. To have a more precise estimation of the damping, a deterministic simulation should be performed for each of the 112 tests. A statistical law could then be provided by the 112 values of damping. However, this approach is very tedious and would ask a lot of simulations. Thus, a few simulations allow estimating the damping of the whirl between $5 \%$ and $11 \%$. This variability could seem high: In practice, with the method employed, the dispersion estimated for the damping could include dispersions of other uncontrolled and unknown parameters. The variability of damping of the whirl instability is introduced with a Gaussian distribution arbitrarily truncated at $\pm \sigma$. Concerning the squeal instability, a damping of $0.5 \%$ is introduced. $10 \%$ is put on the third instability, and $1 \%$ on all the other modes.

A Monte Carlo simulation with 500 samples is performed. The graph of the maximum acceleration of the piston housing is given in Fig. 17(a). The figure makes appear three frequencies: around $235 \mathrm{~Hz}, 470 \mathrm{~Hz}$, and $705 \mathrm{~Hz}$. The first one corresponds to the whirl, which has appeared more precisely between $228 \mathrm{~Hz}$ and $240 \mathrm{~Hz}$. There is a good agreement with the $260 \mathrm{~Hz}$ of the experimental whirl. The two other frequencies are overharmonics of the whirl. It should be noted that the points are not gathered but there is a dispersion of the response between $0 \%$ and $68 \%$ of Gmax. Compared with the experimental results of Fig. 15(a), the graphs are very similar. The maximum acceleration recorded during the tests is of $70 \%$ of Gmax. The dispersion of the response is more precisely illustrated with the pie chart of Fig. 17(b). For more than one-third of the simulations, no vibration or a vibration under $10 \%$ of Gmax appeared. For the tests this proportion reaches more than a half. At the opposite for $15 \%$ of the simulations whirl has appeared with high levels (above $40 \%$ of Gmax). In $16 \%$ of the experimental tests whirl has appeared with similar levels. Moreover, the mean values of acceleration are of $19.8 \%$ of Gmax for the simulations and $17.8 \%$ of Gmax for the tests. There is thus a very good agreement between the Monte Carlo simulation and the experiment.

This result shows that the dispersion observed when a series of tests is performed can be explained with satisfaction by disper- 

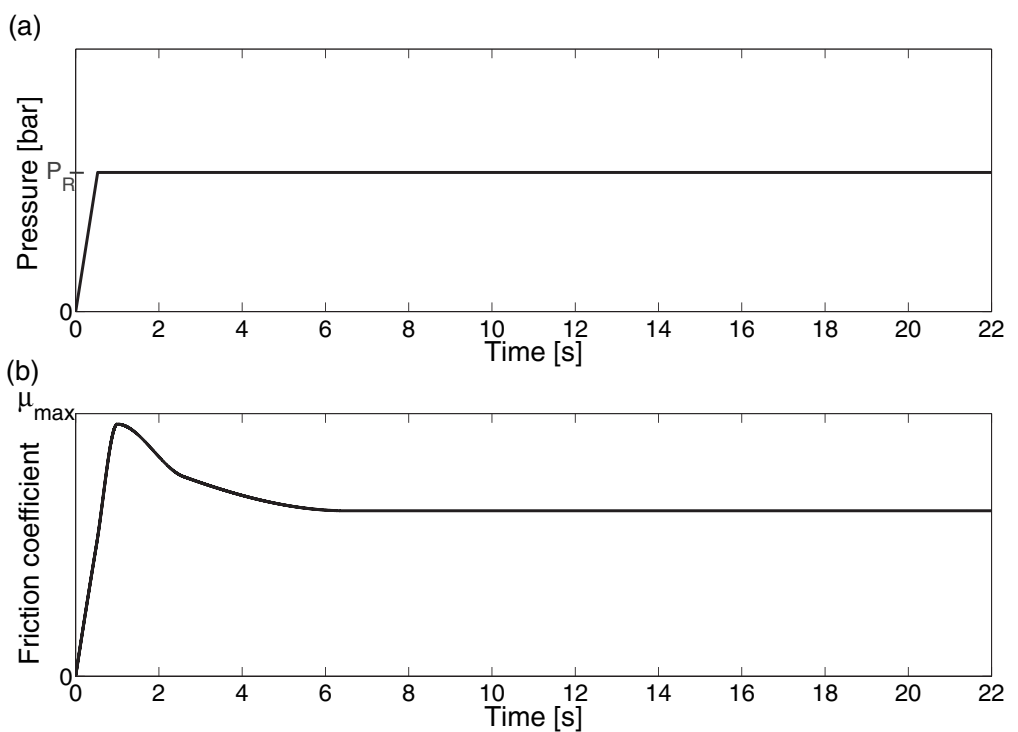

Fig. 11 Simulation: (a) pressure law and (b) friction law

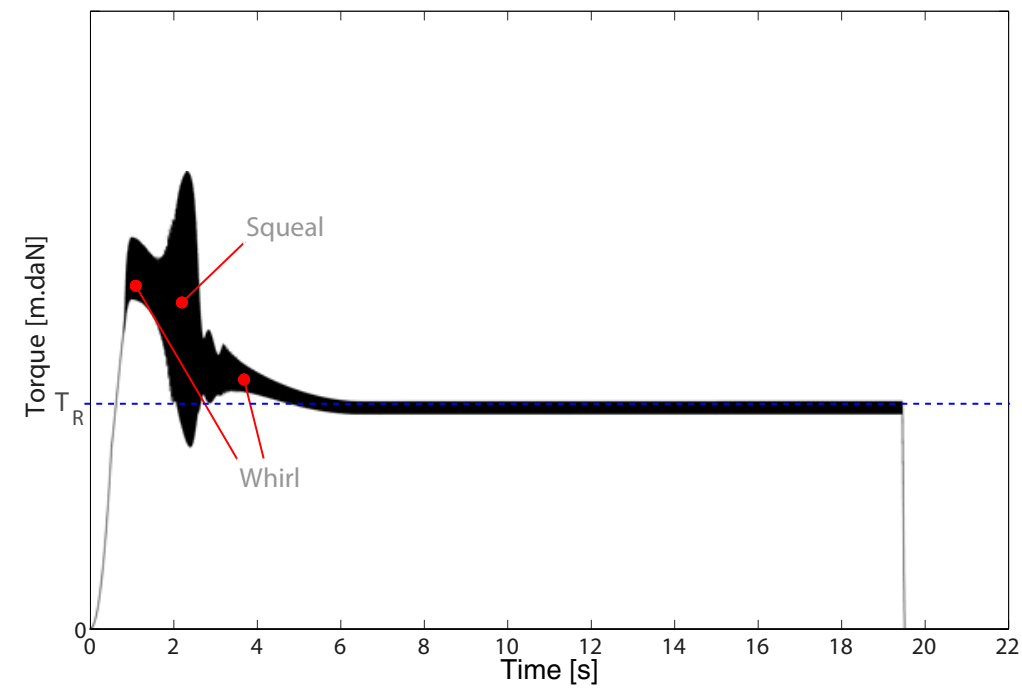

Fig. 12 Deterministic simulation: time plot of the torque

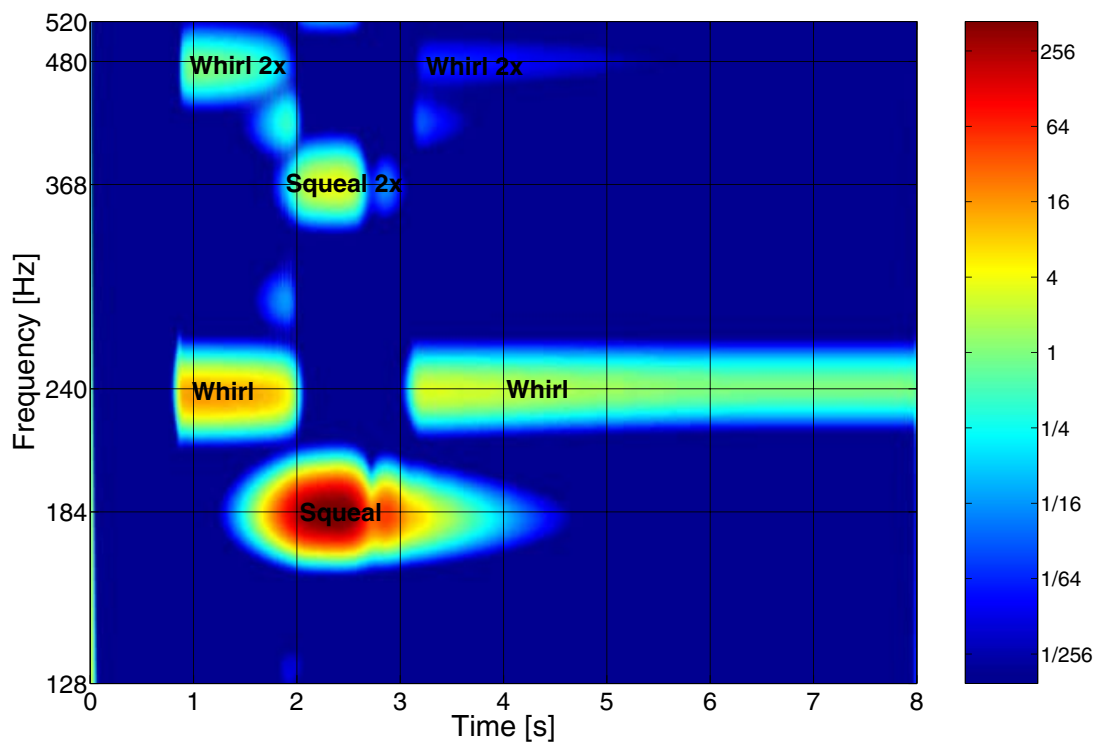

Fig. 13 Deterministic simulation: CWT of the torque 


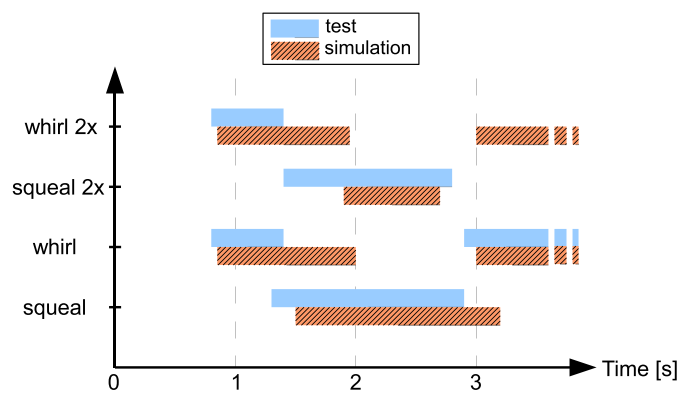

Fig. 14 Deterministic simulation: schematic comparison of the CWT's transient behaviors

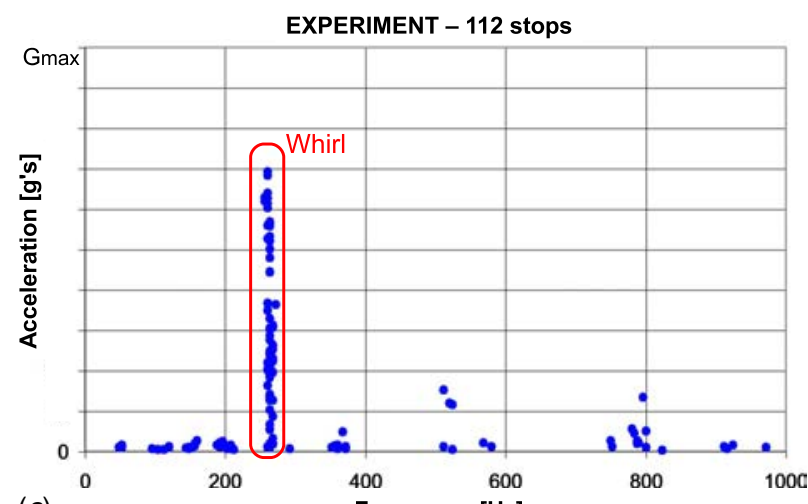

(a)

Frequency $[\mathrm{Hz}]$

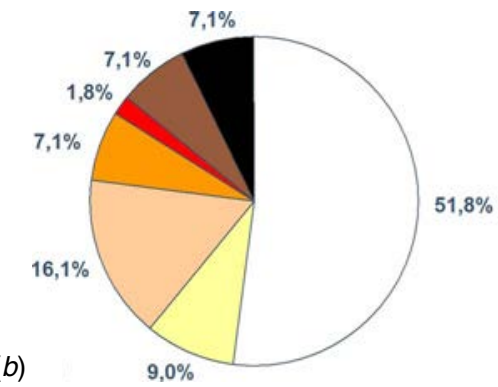

$0 \%$ to $10 \%$ of Gmax $20 \%$ to $30 \%$ of Gmax a $30 \%$ to $40 \%$ of $\mathrm{Gmax}$ - $40 \%$ to $50 \%$ of $G \max$ $50 \%$ to $60 \%$ of $\mathrm{Gmax}$ - $60 \%$ to $70 \%$ of Gmax

Fig. 15 Series of tests: (a) maximum acceleration and (b) pie chart for the whirl

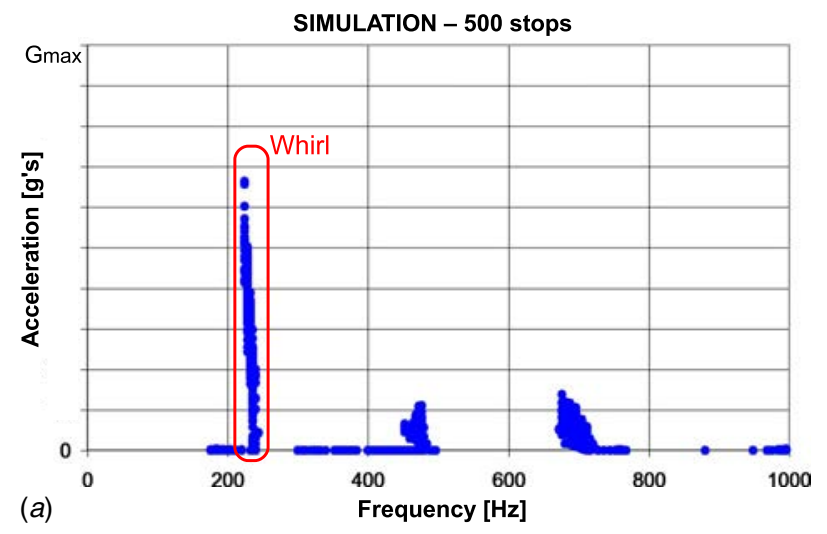

(b)
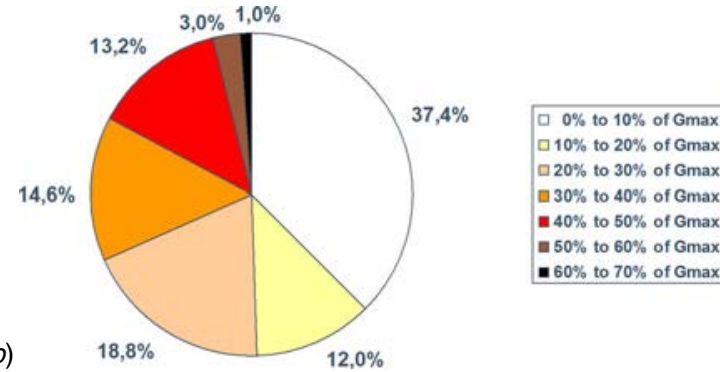

Fig. 17 Monte Carlo simulation: (a) maximum acceleration and $(b)$ pie chart for the whirl

sions in the coefficient of friction and in the damping configuration. The main issue is then to characterize these dispersions. In this study, the use of 112 tests has allowed determining a statistical law for the coefficient of friction. Concerning damping, its measurement on the brake is not directly possible. It has then been approximately estimated with the help of simulations in particular cases. The purposes of this study were to identify the sources of the dispersion of the dynamical response obtained during tests and to evaluate the capability of a phenomenological model to reproduce this dispersion. It would be more complicated to predict the dynamical behavior without having previously performed the tests because the dispersion in the parameters would be unknown. Therefore, in order to perform a predictive simulation, there is still

(a)

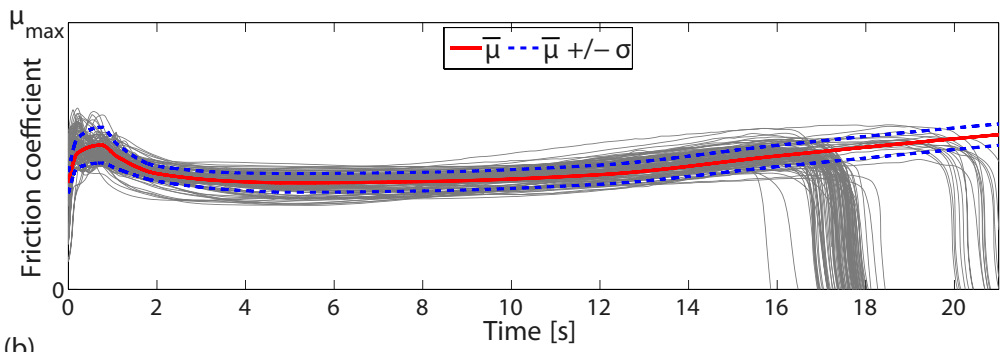

(b)

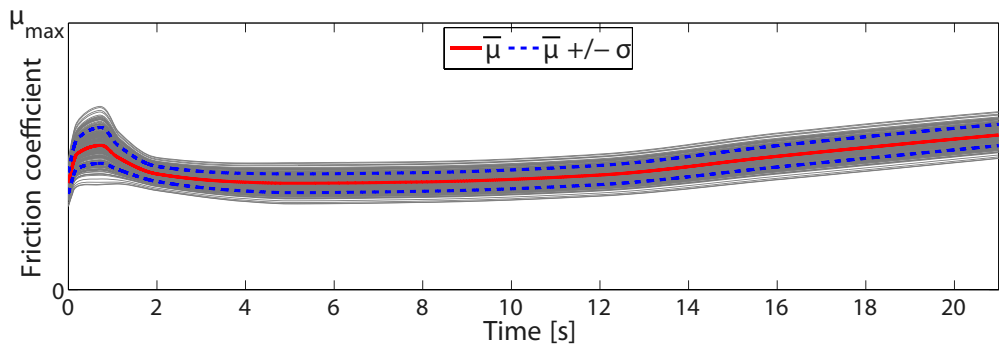

Fig. 16 Friction law: (a) series of test and (b) Monte Carlo simulation 
a lot of work to do on the calculation of the friction coefficient with dispersion and above all on the estimation of the damping in a real complex structure, which is still a great issue.

\section{Summary and Conclusion}

In this paper, a phenomenological model for the simulation of nonlinear vibration induced by friction in aircraft brakes is used to compute time-history responses and to perform a statistical simulation.

From the viewpoint of stability the model that has been used in previous studies is efficient to reproduce squeal and whirl instabilities. The novelty of the present paper concerns the time-history simulation with dispersion of parameters of the dynamical behavior of the system. Provided the friction law and the damping configuration are known, excellent agreements are found between the time-history computation and the experiment. In particular, complex phenomena with interactions between several instabilities are well reproduced.

Tests performed in series show a significant variability in the response of the system. A statistical simulation based on a Monte Carlo method is then employed. The results show that the dispersions of the coefficient of friction and of the damping allow simulating faithfully the dynamical behavior obtained in test. Thus, the causes of the fluctuation in the dynamical behavior are identified and the model allows simulating this fluctuation with a very good agreement. In this context, a predictive simulation requires good knowledge and control of the dispersions of the coefficient of friction and of the damping.

\section{Acknowledgment}

The authors would like to thank Messier-Bugatti for their permission to publish this work.

\section{Appendix: Nonlinear Terms Due to Friction at the Rotor-Stator Interface}

For a point $M$ of radial coordinates $(r, \theta)$, the normal relative displacement $\delta x$ is calculated by considering small displacements of the rotor and the stator

$$
\begin{aligned}
x_{\text {rotor }}(r, \theta)= & x_{r}+r \sin \theta \sin \theta_{r}-r \cos \theta \sin \psi_{r} \approx x_{r}+r \theta_{r} \sin \theta \\
& -r \psi_{r} \cos \theta \\
x_{\text {stator }}(r, \theta)= & x_{s}+r \sin \theta \sin \theta_{s}-r \cos \theta \sin \psi_{s} \approx x_{s}+r \theta_{s} \sin \theta \\
& -r \psi_{s} \cos \theta
\end{aligned}
$$

which give

$$
\begin{aligned}
\delta x(r, \theta)= & x_{\text {stator }}(r, \theta)-x_{\text {rotor }}(r, \theta)=\left(x_{s}-x_{r}\right)+r \sin \theta\left(\theta_{s}-\theta_{r}\right) \\
& -r \cos \theta\left(\psi_{s}-\psi_{r}\right)
\end{aligned}
$$

where $x_{s}, x_{r}, \theta_{s}, \theta_{r}, \psi_{s}$ and $\psi_{r}$ are, respectively, the stator and the rotor lateral displacements and rotations.

Considering the cubic polynomial nonlinear contact stress and the Coulomb law (see Sec. 2.2), the normal force $F_{X}$, the tangential forces $F_{Y}$ and $F_{Z}$, the brake torque $M_{X}$, and the yawing and pitching moments $M_{Y}$ and $M_{Z}$ due to friction can then be expressed as

$$
\begin{aligned}
F_{X}= & \int_{0}^{2 \pi} \int_{R_{i}}^{R_{e}} N(r, \theta) r d r d \theta=\int_{0}^{2 \pi} \int_{R_{i}}^{R_{e}}\left(K_{1} \delta x(r, \theta)+K_{2} \delta x^{2}(r, \theta)\right. \\
& \left.+K_{3} \delta x^{3}(r, \theta)\right) r d r d \theta=K_{1} A_{2}\left(x_{s}-x_{r}\right)+K_{2}\left(A_{2}\left(x_{s}-x_{r}\right)^{2}\right. \\
& \left.+\frac{A_{4}}{4}\left(\theta_{s}-\theta_{r}\right)^{2}+\frac{A_{4}}{4}\left(\psi_{r}-\psi_{s}\right)^{2}\right)+K_{3}\left(A_{2}\left(x_{s}-x_{r}\right)^{3}+\frac{3 A_{4}}{4}\left(\theta_{s}\right.\right.
\end{aligned}
$$

$$
\left.\left.-\theta_{r}\right)^{2}\left(x_{s}-x_{r}\right)+\frac{3 A_{4}}{4}\left(\psi_{s}-\psi_{r}\right)^{2}\left(x_{s}-x_{r}\right)\right)
$$

$$
\begin{aligned}
F_{Y}= & -\int_{0}^{2 \pi} \int_{R_{i}}^{R_{e}} T(r, \theta) \sin \theta r d r d \theta= \\
& -\int_{0}^{2 \pi} \int_{R_{i}}^{R_{e}} \mu_{\mathrm{eq}} N(r, \theta) \sin \theta r d r d \theta=-\mu_{\mathrm{eq}}\left(K_{1} \frac{A_{3}}{3}\left(\theta_{s}-\theta_{r}\right)\right. \\
& +K_{2} \frac{2 A_{3}}{3}\left(\theta_{s}-\theta_{r}\right)\left(x_{s}-x_{r}\right)+K_{3}\left(A_{3}\left(\theta_{s}-\theta_{r}\right)\left(x_{s}-x_{r}\right)^{2}+\frac{3 A_{5}}{20}\left(\theta_{s}\right.\right. \\
& \left.\left.\left.-\theta_{r}\right)^{3}+\frac{3 A_{5}}{20}\left(\theta_{s}-\theta_{r}\right)\left(\psi_{s}-\psi_{r}\right)^{2}\right)\right) \\
F_{Z}= & \int_{0}^{2 \pi} \int_{R_{i}}^{R_{e}} T(r, \theta) \cos \theta r d r d \theta=\int_{0}^{2 \pi} \int_{R_{i}}^{R_{e}} \mu_{\mathrm{eq}} N(r, \theta) \cos \theta r d r d \theta \\
= & -\mu_{\mathrm{eq}}\left(K_{1} \frac{A_{3}}{3}\left(\psi_{s}-\psi_{r}\right)+K_{2} \frac{2 A_{3}}{3}\left(\psi_{s}-\psi_{r}\right)\left(x_{s}-x_{r}\right)+K_{3}\left(A _ { 3 } \left(\psi_{s}\right.\right.\right. \\
& \left.\left.\left.-\psi_{r}\right)\left(x_{s}-x_{r}\right)^{2}+\frac{3 A_{5}}{20}\left(\psi_{s}-\psi_{r}\right)^{3}+\frac{3 A_{5}}{20}\left(\psi_{s}-\psi_{r}\right)\left(\theta_{s}-\theta_{r}\right)^{2}\right)\right)
\end{aligned}
$$

$$
\begin{aligned}
M_{X}= & \int_{0}^{2 \pi} \int_{R_{i}}^{R_{e}} T(r, \theta) r^{2} d r d \theta=\int_{0}^{2 \pi} \int_{R_{i}}^{R_{e}} \mu_{\mathrm{eq}} N(r, \theta) r^{2} d r d \theta \\
= & \mu_{\mathrm{eq}}\left(K_{1} \frac{2 A_{3}}{3}\left(x_{s}-x_{r}\right)+K_{2}\left(\frac{2 A_{3}}{3}\left(x_{s}-x_{r}\right)^{2}+\frac{A_{5}}{5}\left(\theta_{s}-\theta_{r}\right)^{2}\right.\right. \\
& \left.+\frac{A_{5}}{5}\left(\psi_{s}-\psi_{r}\right)^{2}\right)+K_{3}\left(\frac{2 A_{3}}{3}\left(x_{s}-x_{r}\right)^{3}+\frac{3 A_{5}}{5}\left(x_{s}-x_{r}\right)\left(\theta_{s}-\theta_{r}\right)^{2}\right. \\
& \left.\left.+\frac{3 A_{5}}{5}\left(x_{s}-x_{r}\right)\left(\psi_{s}-\psi_{r}\right)^{2}\right)\right)
\end{aligned}
$$

$$
\begin{aligned}
M_{Y}= & \int_{0}^{2 \pi} \int_{R_{i}}^{R_{e}} N(r, \theta) \sin \theta r^{2} d r d \theta=K_{1} \frac{A_{4}}{4}\left(\theta_{s}-\theta_{r}\right)+K_{2} \frac{A_{4}}{2}\left(\theta_{s}-\theta_{r}\right) \\
& \times\left(x_{s}-x_{r}\right)+K_{3}\left(\frac{3 A_{4}}{4}\left(\theta_{s}-\theta_{r}\right)\left(x_{s}-x_{r}\right)^{2}+\frac{3 A_{6}}{24}\left(\theta_{s}-\theta_{r}\right)^{3}\right. \\
& \left.+\frac{3 A_{6}}{24}\left(\theta_{s}-\theta_{r}\right)\left(\psi_{s}-\psi_{r}\right)^{2}\right) \\
M_{Z}= & -\int_{0}^{2 \pi} \int_{R_{i}}^{R_{e}} N(r, \theta) \cos \theta r^{2} d r d \theta=K_{1} \frac{A_{4}}{4}\left(\psi_{s}-\psi_{r}\right)+K_{2} \frac{A_{4}}{2}\left(\psi_{s}\right. \\
& \left.-\psi_{r}\right)\left(x_{s}-x_{r}\right)+K_{3}\left(\frac{3 A_{4}}{4}\left(\psi_{s}-\psi_{r}\right)\left(x_{s}-x_{r}\right)^{2} \frac{3 A_{6}}{24}\left(\psi_{s}-\psi_{r}\right)^{3}\right. \\
& \left.+\frac{3 A_{6}}{24}\left(\psi_{s}-\psi_{r}\right)\left(\theta_{s}-\theta_{r}\right)^{2}\right)
\end{aligned}
$$

with $A_{k}=\pi\left(R_{e}^{k}-R_{i}^{k}\right)$ for $k=2-6$ and $R_{e}$ and $R_{i}$, respectively, the outer and inner radii of the contact surface.

\section{References}

[1] Spurr, R., 1961, “A Theory of Brake Squeal," Proc. Auto. Div. Instn. Mech. Engrs, 1, pp. 33-40.

[2] Crolla, D. A., and Lang, A. M., 1991, "Brake Noise and Vibration-The State of Art," Vehicle Tribology, 18, pp. 165-174.

[3] Kinkaid, N., O'Reilly, O., and Papadopoulos, P., 2003, "Automotive Disc Brake Squeal," J. Sound Vib., 267, pp. 105-166.

[4] Moirot, F., 1998, "Etude de la stabilité d'un équilibre en présence de frotte- 
ment de Coulomb. Application au crissement des freins à disque," Ph.D. thesis, Ecole Polytechnique, Palaiseau, France.

[5] Drobecq, V., 1997, "Modélisation de systémes de freinage ferroviaires avec prise en compte du phénomène de crissement," Ph.D. thesis, Université de Valenciennes, Valenciennes, France.

[6] Lorang, X., 2007, "Instabilité vibratoire des structures en contact frottant. Application au crissement des freins de TGV," Ph.D. thesis, Ecole Polytechnique, Palaiseau, France.

[7] Liu, S., Ozbek, M., and Gordon, J., 1996, "A Nonlinear Model for Aircraft Brake Squeal Analysis. Part I: Model Description and Solution Methodology," ASME Design Engineering Technical Conferences, Vol. 3, pp. 406-416.

[8] Liu, S., Ozbek, M., and Gordon, J., 1996, "A Nonlinear Model for Aircraft Brake Squeal Analysis. Part II: Stability Analysis and Parametric Studies,' ASME Design Engineering Technical Conferences, Vol. 3, pp. 417-425.

[9] Gordon, J., 1997, "A Perturbation Analysis of Nonlinear Squeal Vibrations in Aircraft Braking Systems," ASME Design Engineering Technical Conferences, Sacramento, CA, Sept. 14-17.

[10] Travis, T., 1995, "Nonlinear Transient Analysis of Aircraft Landing Gear Brake Whirl and Squeal," ASME Design Engineering Technical Conference 3 , Vol. 84-1, Pt. A.

[11] Hagler, L. B., and Reinhall, P. G.1997, "Friction Induced Vibrations in Aircraft Disk Brakes,” ASME Design Engineering Technical Conferences, Sacramento, CA, Sept. 14-17.
[12] Sinou, J.-J., Dereure, O., Mazet, G.-B., Thouverez, F., and Jézéquel, L., 2006 "Friction Induced Vibration for an Aircraft Brake System. Part 1: Experimental Approach and Stability Analysis," Int. J. Mech. Sci., 48, pp. 536-554.

[13] Sinou, J.-J., Thouverez, F., Jézéquel, L., Dereure, O., and Mazet, G.-B., 2006, "Friction Induced Vibration for an Aircraft Brake System. Part 2: Non-Linear Dynamics," Int. J. Mech. Sci., 48, pp. 555-567.

[14] Chevillot, F., Sinou, J.-J., Mazet, G.-B., Hardouin, N., and Jézéquel, L., 2008, "The Destabilization Paradox Applied to Friction-Induced Vibrations in an Aircraft Braking System," Arch. Appl. Mech., 78, pp. 949-963.

[15] Chevillot, F., Sinou, J.-J., Hardouin, N., and Jézéquel, L., 2009, "Nonlinear Transient Vibrations and Coexistences of Multi-Instabilities Induced by Friction in an Aircraft Braking System," J. Sound Vib., 328, pp. 555-574.

[16] Sinou, J.-J., 2009, "An Experimental Investigation of Condition Monitoring for Notched Rotors Through Transient Signals and Wavelet Transform," Exp. Mech., 49, pp. 683-695.

[17] Morlet, J., 1983, Sampling Theory and Wave Propagation, Issues in Acoustic Signal/Image Processing and Recognition, NATO ASI Series, C. H. Chen, ed., Springer-Verlag, Berlin, Vol. I, pp. 233-261.

[18] Mallat, S., 1998, A Wavelet Tour of Signal Processing, Academic, New York

[19] Louis, A., Maaß, P., and Rieder, A., 1994, Wavelets, B.G. Teubner, Leipzig.

[20] Chui, C., 1992, An Introduction to Wavelets, Academic, New York. 